\title{
III.
}

\section{Bericht über die im Jahre 1879 in meiner Poliklinik für Ohrenkranke beobachteten Krankheitsfälle.}

\author{
Von
}

\author{
Dr. K. Bürkner \\ in Göttingen.
}

In der Zeit vom 1. Januar bis 31. December 1879 haben sich - abgesehen von 20 Restanten aus dem Vorjahre - 328 Patienten mit 359 verschiedenen Krankheitsformen vorgestellt. Davon wurden 305 in Behandlung genommen, 23 nach ein- oder mehrmaliger Untersuchung als unheilbar abgewiesen; bei $5 \mathrm{~Pa}$ tienten konnte, theils wegen Ausbleibens nach der ersten zufällig unvollständigen Untersuchung, theils wegen Unzulänglichkeit meiner Notizen, keine genaue Diagnose gestellt werden.

Geheilt wurden . . . . . . . 148 oder 45,2 Proc.

Wesentlich gebessert . . . . . . . $69,21,0$ n

Ungeheilt blieben . . . . . . . . 18 " 5,4 "

Ohne Behandlung entlassen wurden . . 23 , 7,1,

Der Erfolg der Behandlung blieb unbe-

kannt, weil die Patienten ausblieben bei 42 , 12,8 n

Gestorben ist . . . . . . . . 1 , 0,3 "

In Behandlung verblieben $\cdot \cdot \cdot \frac{27 n \frac{8,2}{3}}{328}$

Als wirklicher Procentsatz der Geheilten nach Abzng der noch in Behandlung befindlichen und nicht in Behandlung genommenen Patienten ergibt sich 53,2 Proc., für die geheilten und gebesserten Fälle zusammen 78,0 Proc.

Von den 328 Patienten waren

aus Göttingen: 123 , d. i. 37,5 Proc.

von auswärts: 205 , d. i. 62,5 "

Männlichen Geschlechts waren 212, d. i. 64,6 Proc.

Weiblichen " " 116, d. i. 35,4 "

Kinder waren . . . . 94, d. i. 29,9 "

Erwachsene . . . 234, d. i. 71,1 , 
Bericht üb. d. in meiner Polikl. f, Ohrenkr. beobacht. Kranksheitsfäle. 1879. 57

Auf die verschiedenen Krankheiten vertheilen sich die einzelnen Fälle in folgender Weise.

\begin{tabular}{|c|c|c|c|c|c|c|c|c|}
\hline Nomen thorbi & $\frac{\mathscr{E}}{\tilde{E}}$ & 咅 & 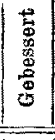 & 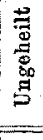 & 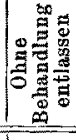 & 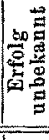 & 醇 & 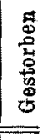 \\
\hline Tumor auris extern. . . . . . . . . & 2 & - & - & - & 1 & 1 & - & - \\
\hline $\begin{array}{l}\text { Eczema auris ext. Acut. } 6 \text {, chron. } 4 \text {, ein- } \\
\text { seitig } 3 \text {, doppels. } 7 \text {. } \dot{5} \text {, chron. } 5 \text {, ein- }\end{array}$ & 10 & 6 & - & 1 & - & 3 & - & - \\
\hline $\begin{array}{l}\text { seitig } 10 \text {, doppels. } 4 \text {. } \\
\text { Otitis externa circumscripta. Einseitig } i \text {, }\end{array}$ & 14 & 10 & - & - & - & 3 & 1 & - \\
\hline $\begin{array}{l}\text { doppelseitig } 1 \\
\text { Accumulatio ceruminis, Einseitig } 18 \text {, doppel- } \\
\text { seitig } 25\end{array}$ & 43 & 34 & - & - & - & - & - & - \\
\hline Corpus alienum. Einseitig. 3 , doppelseitig i & 4 & 4 & - & - & - & - & - & - \\
\hline Myringitis acuta. Einseitig 6 , doppelseitig 1 & 7 & 7 & - & - & - & - & -.. & - \\
\hline $\begin{array}{l}\text { Myringitis chronica. Einseitig 3, doppels. } 0 \\
\text { Traumatische Trommelfellaffection. Einseitig }\end{array}$ & 3 & 1 & 2 & - & - & - & - & - \\
\hline $\begin{array}{l}7 \text {, doppelseitig } 0 \\
\text { Otitis med simpl. acuta. Einseitig } 2 \text {, dop- }\end{array}$ & 7 & 7 & - & - & - & - & - & - \\
\hline & 18 & 17 & 1 & - & - & - & - & - \\
\hline & 90 & 18 & 32 & 6 & $\mathfrak{5}$ & 17 & 12 & - \\
\hline $\begin{array}{l}\text { Salpingitis acuta. Einseitig 1, doppelseit. } 7 \\
\text { Salpingitis chronica. Einseitig 1, doppelseit. }\end{array}$ & 8 & 7 & - & - & - & 1 & 7 & - \\
\hline $\begin{array}{l}\text { Salpingitis chronica. Einseitig } 1 \text {, doppelseit. } 2 \\
\text { Otitis med. suppur. acuta. Einseitig 10, dop- }\end{array}$ & 3 & 1 & - & - & - & - & 2 & - \\
\hline $\begin{array}{l}\text { pelseitig } 1 \\
\text { otitis med. suppur. chron. Einseitig } 26 \text {, dop- }\end{array}$ & 11 & 8 & 1 & - & - & - & 1 & - \\
\hline $\begin{array}{l}\text { pelseit. } 29 . \text { Polypi } 11 \text {, Caries } 6 \\
\text { Labyrinth-Nervenaffection acut. Einseitig } 0 \text {, }\end{array}$ & 55 & 15 & 17 & 1 & - & 13 & 8 & 1 \\
\hline $\begin{array}{l}\text { doppelseitig } 4 \\
\text { Labyrinth-Nervenatfection chronisch. Ein- }\end{array}$ & 4 & 1 & 1 & - & - & 2 & - & - \\
\hline $\begin{array}{l}\text { seitig } 7 \text {, doppelseitig } 6 \\
\text { Taubstummheit. Erworben } 4 \text {, angeboren } i\end{array}$ & $\begin{array}{r}13 \\
5\end{array}$ & 二 & $\overline{-}$ & $\begin{array}{l}4 \\
1\end{array}$ & $\begin{array}{l}7 \\
2\end{array}$ & $\overline{1}$ & $\begin{array}{l}2 \\
1\end{array}$ & 二 \\
\hline Periostitis proc. mast. . . . . . & 1 & 1 & - & - & - & - & - & - \\
\hline $\begin{array}{l}\text { Parotitis } \\
\text { Abgelaufene Mittelohrprocesse Einseitig } 9 \text {, }\end{array}$ & 1 & 1 & - & - & - & - & - & - \\
\hline 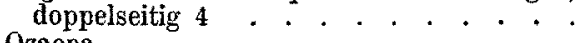 & 13 & 2 & 6 & 3 & 1 & 1 & - & - \\
\hline 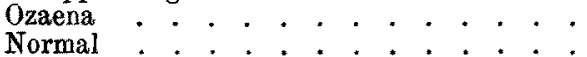 & 1 & $=$ & - & 1 & $\overline{2}$ & - & - & - \\
\hline Keine Diagnose & 5 & - & - & - & 5 & - & - & - \\
\hline & & & & 18 & 23 & 42 & 27 & 1 \\
\hline
\end{tabular}

Es kamen somit auf die

Krankheiten des äusseren Obres 81

\begin{tabular}{|c|c|c|c|}
\hline$n$ & $"$ & Trommelfelles & 17 \\
\hline$n$ & $n$ & Mittelohres & 198 \\
\hline$n$ & $n$ & inneren Obres & 17 \\
\hline Diverses &. & . . . . . & 15 \\
\hline
\end{tabular}

Von den 359 verschiedenen, das Ohr betreffenden Krankheitsformen waren doppelseitig 229 oder 63,8 Proc., einseitig 130 oder 
36,2 Proc., und von den einseitigen betrafen 47 (36,1 Proc.) das rechte, 83 (63,9 Proc.) das linke Ohr.

Hervorzuheben wären etwa folgende Fälle:

1 Fall ron Fibrom des äusseren Obres; eine halbkugelige harte, aber elastische Geschwulst füllte die Concha eines 5 jährigen Knaben fast gänzlich aus; da der Kranke ausblieb, war die Diagnose nicht mit voller Sicherheit zu stellen. - Bei einem 20 jährigen Studenten fand ich $1 \mathrm{Mm}$. unterhalb der Ohrmuschel eine gleichfalls halbkugelige, ziemlich harte, mit nicht verschiebbarer Haut bedeckte, aber selbst etwas verschiebbare Geschwulst von circa $6 \mathrm{Mm}$. Radius zwischen Warzenfortsatz und Unterkieferwinkel; leider blieb auch dieser Patient nach einmaliger Untersuchung aus; wahrscheinlich war die Geschwulst ein Lipom.

Unter den Fällen von Fremdkörpern verdient ein Fall Erwähnung, welcher einen 24 jährigen Schneidergesellen betraf; demselben war iber Nacht ein Ohrwurm (Forficula auricularia) in das rechte 0 hr gekrochen und verursachte durch seine Betastungen des Trommelfelles, das sehr stark injicirt war, ungemein heftige Schmerzen.

Unter den Trommelfellbefunden wurden besonders notirt: Verkalkungen 23 mal, Narben 25 mal, Verkalkungen mit Narben combinirt 8 mal, Ekehymosen 9 mal, Pigmentir ung 1 mal, Cholesteat ome in Gestalt kleiner Perlen 1 mal.

Ein verhältnissmässig recht beträchtliches Contingent (7) stellten die traumatischen Trommelfellaffectionen. Dreimal war ein Schlag auf das Obr als Ursache zu verzeichnen; in einem von diesen wurde das Trommelfell imperforirt mit den Gehörknöchelchen nach innen gedrückt, wodurch hochgradige Hirnerscheinungen veranlasst wurden, in den beiden anderen war es zu Durchreissungen und Ekchymosen gekommen. Zweimal handelte es sich um einfache Perforation mit spitzen Instrumenten (Stricknadel und Haarnadel), einmal hatte sich die Patientin nicht allein das Trommelfell mit einer Stricknadel durchstochen, sondern auch den Ambosschenkel oder den Steigbügel getroffen; mehrtägige Schwindelerscheinungen und Erbrechen waren die Folgen; und schliesslich hatte ein Mädchen sich beim Ausweichen auf schmalem Wege das Trommelfell an einem Aestchen einer Hecke gespiesst. Sämmtliche Fälle wurden geheilt.

Unter den Fällen von chronischen Mittelohreiterungen verliefen einige recht stürmisch; 4 mal kam es zu meningitischen 
Symptomen, doch wurden 3 von diesen Patienten gerettet, während einer starb. Es war dies eine 44 jährige Bänerin ans Geismar, die angeblich seit wenigen Tagen unter heftigen Schmerzen an Ausfluss aus dem linken Ohre litt. Der Gehörgang war so stark gesehwollen, dass das Secret nur eben abfliessen konnte; als ich die Kranke das erste Mal sah, fieberte sie bereits; am nächsten Tage war nach Anwendung von kalten Umschlägen und Borsäure einige Besserung eingetreten; aber schon wenige Tage später fand ich bei meinem Besuche die Patientin sehr collabirt, uiber Schmerzen im Nacken und beim Schlingen im Halse klagend, in hohem Fieber, das mit mehreren Schüttelfrösten täglich einherging; der Gehörgang war abgeschwollen, die Eiterung stand, das Trommelfell zeigte sich vorn unten perforirt; der Warzenfortsatz war nicht im geringsten sehmerzhaft. 8 Tage später war die Gegend zwischen linkem Sternocleidomastoideus und Wirbelsäule geschwollen, sehr bart, äusserst schmerzhaft, die Halsdrisen zeigten sich infiltrirt, so dass die Diagnose auf $\mathrm{Phlebitis} \mathrm{des}$ Sinus mit Sicherheit gestellt werden konnte. 3 Tage darauf war die Kranke nach einem mehrstündigen comatösen Stadium todt. Section konnte nicht ausgeführt werden.

Unter den Fällen von Krankheiten des Warzenfortsatzes, die als Complicationen beobachtet wurden, waren zwei von besonderem Interesse. Der eine betraf eine 36 jährige Frau, welche wegen einer durch ausgedebnte Vernarbungen bedingten Schwerhörigkeit Hiilfe suchte. Hier zeigte sich hinter dem rechten Ohre auf dem Planum mastoideum eine Grube von $0,5 \mathrm{Cm}$. Durchmesser und $1,25 \mathrm{Cm}$. Tiefe, fast genau parallel mit dem Gehörgange nach innen verlaufend und mit bröckeligen, braunen Massen ausgeftullt; der Grund des Kanales war elastisch, die Speculation zeigte eine weissliche glänzende Haut. Auf meine Frage, ob diese Vertiefung von einer Operation herrühre, wurde mir geantwortet, dass bei einer Eiterung in der Kindheit eine Fistel am Warzentheile entstanden sei, die künstlich erweitert wurde. Beim Ausspritzen floss kein Wasser aus dem Ohre ab.

Der 2 weite Fall betraf einen 21 jährigen jungen Mann aus der Gegend von Minden, aus dessen Angaben hervorging, dass er als 6 jähriger Knabe durch einen Steinwurf hinter dem rechten Ohre verletzt worden war; in der Wunde hatte sich am Tage nach der Verletzung eine kleine Blase gezeigt, welche von der Mutter des Kranken mit der Stopfnadel durchstochen worden war; dabei war die Nadel tief in den Knochen bineingerathen. 
Ein zugezogener Arzt hatte das Loch mit Pressschwamm erweitert und für Offenhaltung gesorgt. Als der Kranke in meine Behandlung trat, zeigte sich hinter dem rechten Obre, circa in der Höhe des obersten Drittels der Muschel, ein 6,5 Mm. weites Loch, aus dem ein höchst fötider Eiter tropfte, obgleich die Höhlung im Knochen über $1 \mathrm{Cm}$. unter die Oeffnung hinabreichte. Nach dem Ausspritzen, das viel Eiter und abgestossene Haut, sowie mebrere halbmacerirte Zwirnpfropfen zu Tage förderte, mit denen er das Loch verstopft hatte, gelang es mit der Sonde nach vorn eirca $1 \mathrm{Cm}$., nach hinten reichlich $3 \mathrm{Cm}$, nach innen $1,5-2 \mathrm{Cm}$. weit einzudringen, ohne dass dem Kranken wesentliche Schmerzen verursacht wurden; die Wände des Hohlraumes waren zum grossen Theile mit glatter und, so weit zu beleuchten, weisser Haut ausgefüllt, nur vorn und hinten stiess die Sonde auf cariösen Knochen. Beim Spritzen wurde der Kranke sehr schwindlig, ebenso bei stärkerem Drucke der Sonde auf die innere und obere Wand; ans dem Ohre floss nicht ein Tropfen Flussigkeit $a b$; das Trommelfell war bis auf eine gleichmässige, graue Triibung vollständig normal, die Luftdouche ergab normales Anschlagegeräusch, die Uhr wurde auf mehrere Centimeter gehört, Sprache fast ganz gut.

Vor Allem wurden regelmässige Ausspülungen mit Carbolsäurelösung verordnet, bei dem nächsten Besuche des Patienten zuerst mit der Sonde, dann mit einem kleinen' scharfen Löffel einige cariöse Knochenmassen entfernt und diese Operation noch zweimal wiederholt. Im Laufe der nächsten Monate hörte die Eiterung vollkommen anf, die Ueberhäutung schritt vorwärts and das Gehör wurde gebessert. Wie weit die noch im Gange befindliche Behandlung zur Heilung fubren wird, ist freilich noch nicht abzusehen, da sich bis vor 4 Wochen noch keine Granulationen gebildet hatten.

Unter den im verflossenen Jahre in Anwendung gezogenen Arzneimitteln stand oben an die Borsäule, die ich seit dem Mai in verschiedener Form verordnet habe. Besonders bei Eezem und Obreneiterungen wirkte das Mittel mitunter uberraschend gut. Bei Eczem zog ich die Salbenform (2,5 und 5,0:20-30 Vaselin) vor, während ich die Eiterung in der Regel mit Lösung und Pulver behandelte.

Schliesslich spreche ich noch den Herren Dr. Hauptmann und Dr. W engler für ihre Unterstützung während der Zeit der grössten Frequenz meinen Dank aus. 